\title{
Three-Tesla MR imaging of the elbow in non-symptomatic professional baseball pitchers
}

\author{
Filippo Del Grande • Michael Aro • Sahar Jalali Farahani • \\ John Wilckens • Andrew Cosgarea • John A. Carrino
}

Received: 25 June 2014 /Revised: 3 September 2014 / Accepted: 19 September 2014 / Published online: 10 October 2014

(C) ISS 2014

\begin{abstract}
Objectives To retrospectively evaluate the qualitative and quantitative 3-T MR imaging features of the elbow in nonsymptomatic professional baseball pitchers presenting as major league draft picks or trades.

Materials and methods The Institutional Review Board (IRB) approved the HIPPA-compliant study. Informed consent was waived. Twenty-one professional non-symptomatic baseball pitchers (mean age 23, range 18 to 34 years old) underwent 3T MR imaging of the pitching elbow. Two experienced readers independently performed qualitative (collateral ligaments, tendons, cartilage, bones, ulnar nerve, olecranon fossa, and joint fluid) and quantitative (collateral ligaments and posteromedial plica) evaluation. Descriptive statistics were calculated.

Results Collateral ligament thickening was seen in a high proportion, nearly half, however, without features of full thickness tearing. Tendinosis without tearing was seen in $19 \%(4 / 21)$ of common extensors. Cartilage abnormalities were infrequent. Bone abnormalities manifested as edema in $24 \%(5 / 21)$ and humeroulnar osteophytosis. Ulnar nerve signal and/or morphologic abnormalities were seen in a very high proportion, up to $81 \%(17 / 21)$. The olceranon fat pad showed scarring features in about one third. The median
\end{abstract}

F. Del Grande $\cdot$ M. Aro $\cdot$ S. J. Farahani · J. A. Carrino

The Russell H Morgan Department of Radiology and Radiological

Sciences, Johns Hopkins University School of Medicine, 601 North

Wolfe Street, Baltimore, MD 21287, USA

F. Del Grande ( $\triangle)$

Ospedale Regionale di Lugano, Servizio di Radiologia. Lugano, Via

Tesserete. 6900, Lugano-TI, Switzerland

e-mail: fdelgra1@jhmi.edu

J. Wilckens $\cdot$ A. Cosgarea

Department of Orthopaedic Surgery, Johns Hopkins Hospital,

Baltimore, MD, USA ligament thicknesses in mm measured: 4.6 UCL anterior bundle, 1.8 UCL posterior bundle, 1.9 RCL, 2.5 LUCL, and $0.7 \mathrm{~mm}$ anular. The median plica dimensions were 5.3 by 2.2 by $2.7 \mathrm{~mm}$.

Conclusion High-resolution 3-T MR imaging frequently shows abnormalities involving the ligaments, tendons, nerves, olecranon fat pad, and bones in non-symptomatic baseball pitchers.

Keywords Magnetic resonance imaging - Sports medicine · Elbow

\section{Introduction}

Elbow MR imaging plays a key role in the diagnosis of sports medicine elbow pathologies and may be performed on nonsymptomatic professional baseball players before contract signing. Repetitive micro-trauma during pitching leads to morphological changes on MR imaging seen even in nonsymptomatic professional baseball players [1]. During pitching, the elbow joint is submitted to high valgus stress forces $[2,3]$. Compressive forces in the lateral compartment and tensile forces in the medial compartment can lead to the clinical picture of "valgus extension overload syndrome." High-field 3-T MR imaging is increasingly being employed in clinical sports medicine practice for high-resolution joint imaging, owing to the higher signal-to-noise ratio (SNR) and improved image quality particularly for smaller joints [4]. Hence, it is important to be aware of the MR imaging findings that may be encountered in non-symptomatic baseball pitchers on 3-T MR imaging to avoid false-positive results and misdiagnosis. Therefore, our purpose was to retrospectively evaluate the qualitative and quantitative 3-T MR imaging features of the elbow in non-symptomatic professional baseball pitchers presenting as major league draft picks or trades. 


\section{Material and methods}

Subjects

This retrospective study was approved by the Institutional Review Board of our institution and was HIPPA compliant. Informed consent was waived. We selected non-symptomatic professional baseball pitchers that were draft picks or trades of the local professional baseball team who underwent 3-T elbow MR imaging performed at our institution between February 2008 and August 2011. Every pitcher underwent physical examination performed by an experience orthopedic surgeon trained in sports medicine. All included pitchers claimed to be non-symptomatic. All subjects were negative for pain on the medial and lateral elbow, milking maneuver, and on valgus stress maneuver. One subject had some mild laxity but no discomfort on the valgus stress maneuver. Exclusion criteria were: previous surgery on the elbow (1 subject excluded) and incomplete/insufficient quality of the MR imaging (1 subject excluded). According to these criteria, we included 21 male subjects with a mean age of 23 years (range 18-34 years old), 4 left hand dominant and 17 right hand dominant. Medical records were available to the examining physician.

\section{MR imaging protocol}

MR imaging was performed on a wide-bore 3-T MR system (MAGNETOM Verio, Siemens Medical Solutions, Erlangen, Germany). The subjects were imaged in supine position with the arm at the side in a neutral position. The images were acquired with an eight-channel receiver phased-array flexible surface coil. MR sequences and parameters are the following: T1-weighted turbo spin-echo sequences in the transverse plane (repetition time ms/echo time ms, 570-733/11-14; section thickness, $3 \mathrm{~mm}$; distance factor, $26 \%$; field of view, 130 $\times 130$; matrix size, $256 \times 256$; voxel size, $0.5 \times 0.5 \times 3 \mathrm{~mm}$; acquisitions 1; iPAT 2); intermediate-weighted sequences in the transverse plane (repetition time ms/echo time ms, 3,0003,350/28-45; section thickness, $3 \mathrm{~mm}$; distance factor, $10 \%$; field of view, $130 \times 130$; matrix size, $256 \times 256$; voxel size, $0.5 \times 0.5 \times 3 \mathrm{~mm}$; acquisitions 2 ; iPAT 2); intermediateweighted sequences, fat suppressed with spectral adiabatic inversion recovery (SPAIR) in the transverse plane $(2,450$ 3,790/28-72; section thickness, $3 \mathrm{~mm}$; distance factor, $15 \%$; field of view, $120 \times 120$; matrix size, $256 \times 256$; voxel size, $0.5 \times 0.5 \times 3 \mathrm{~mm}$; acquisitions 1 ; iPAT off); intermediateweighted sequences in coronal plane (2,200-3,000/36-46; section thickness, $3.5 \mathrm{~mm}$; distance factor, $10 \%$; field of view, $130 \times 130$; matrix size, $256 \times 256$; voxel size, $0.5 \times 0.5 \times$ $3.5 \mathrm{~mm}$; acquisitions 3; iPAT 2); intermediate-weighted sequences, fat suppressed with SPAIR in coronal planes $(2,000$ 4,500/42-49; section thickness, $3.5 \mathrm{~mm}$; distance factor, $10 \%$; field of view, $130 \times 130$; matrix size, $256 \times 256$; voxel size, $0.5 \times 0.5 \times 3.5$; acquisitions 2 ; iPAT off); T2-weighted sequences in sagittal planes $(3,890-4,810 / 49-82$; section thickness, $3.0 \mathrm{~mm}$; distance factor, $10 \%$; field of view, 130 $\times 130$; matrix size, $256 \times 256$; voxel size, $0.5 \times 0.5 \times 3.0 \mathrm{~mm}$; acquisitions 2; iPAT 2); intermediate-weighted sequences, fat suppressed (SPAIR) in the sagittal plane (2,000-4,360/42-49; section thickness, $3.0 \mathrm{~mm}$; distance factor, $10 \%$; field of view, $130 \times 130$; matrix size, $256 \times 256$; voxel size, $0.5 \times 0.5 \times 3.0$; acquisitions 3; iPAT 2).

\section{Analysis of MR imaging}

Images were evaluated independently by three radiologists (reader 1 with 12 years of experience in musculoskeletal MR imaging; reader 2 with 10 years of experience of musculoskeletal MR imaging; reader 3 with 10 years of experience of musculoskeletal MR imaging). Qualitative imaging evaluation was performed by readers 1 and 2 independently. Subsequently, a consensus reading was performed. Quantitative evaluation was performed by reader 2 and reader 3 independently. All images were reviewed on a PACS workstation (Ultravisual, Merge Healthcare, Chicago, IL, USA) with diagnostic quality monitors.

\section{Qualitative evaluation}

Two readers independently evaluated the following elbow structures/features: ligaments, tendons, cartilage, cubital tunnel, osteophytes, joint fluid, olecranon fat pad, bone marrow, and intra-articular loose bodies. The MR imaging findings for each structure were constructed as ordinal variables.

Ligaments: The ligaments were evaluated for integrity (intact, partial tear less than $50 \%$ of the thickness, partial tear more than $50 \%$ of the thickness, full thickness tear) and for tear location (proximal attachment, mid-portion, distal attachment). Furthermore, ligaments were evaluated for signal intensity on fluid-sensitive sequences (normal, i.e., homogeneous low signal intensity or hyperintense to surrounding muscles) and for signal intensity patterns such as striation (heterogeneous signal pattern) according to Husarik et al. [5].

Tendons: The common flexor tendon, common extensor tendon, and biceps tendon were evaluated as normal (homogeneous low signal intensity), tendinosis (high signal intensity less than fluid), partial tear (fluid-like signal intensity with continuity of the tendon), or full thicknesses tear (loss of continuity of the tendon).

Bones: Osteophytes were documented if present and characterized as small, moderate, and large in the humeroulnar (with special note if posteromedial), humeroradial (radiocapitellar), and proximal radioulnar joint. Small osteophytes were defined as less than $2 \mathrm{~mm}$, medium osteophytes between 2 and $5 \mathrm{~mm}$, and large osteophytes more 
than $5 \mathrm{~mm}$. Bone marrow edema was recorded if present and the location documented.

Cartilage: We evaluated the humeroulnar, humeroradial, and proximal radioulnar joints for cartilage lesions, focal defects, flaps, or diffuse thinning. A diffuse lesion was defined as a lesion of more than $5 \mathrm{~mm}$ in at least one plane.

Cubital tunnel: Defined as the fibroosseous channel formed by the olecranon process laterally, the posterior cortex of the medial epicondyle medially, the elbow joint capsule and posterior bundle of the medial collateral ligament anteriorly, and the cubital retinaculum posteriorly. The ulnar nerve was evaluated within the cubital tunnel for signal intensity (isointense to adjacent muscle, slightly higher signal intensity than muscle, moderately higher signal intensity but less than the vessel, and signal intensity the same as the adjacent vessel/vein) following the same criteria as Chhabra and colleagues [6] and for cross-sectional shape (ovoid or flattened) within the cubital tunnel. A flattened ulnar nerve was defined as a greater than 2 to 1 ratio between the width and height. The cubital retinaculum was evaluated for presence and thickness (absent, normal, or thickened). A thickened retinaculum was defined as other than a very thin linear configuration.

Joint fluid: The joint cavity was evaluated for effusion and then graded as none (no distension of the anterior and posterior elbow recess), small (distension of the anterior recess), or large (distention of the anterior and posterior recess) and for the presence of intra-articular bodies (if any).

Olecranon fat pad: This feature was defined as normal, acute, or chronic changes. Acute changes were considered when edema (fluid-like signal) was present with low signal intensity on T1-weighted sequences and high signal intensity on fluid-sensitive sequences. Chronic changes were considered when fibrosis (fibrous-like signal) was present with low (dark) signal intensity on T1-weighted and also low (dark) signal intensity on fluid-sensitive sequences.

\section{Quantitative evaluation}

The thickness of the ligaments [anterior bundle of the ulnar collateral ligament (UCL), posterior bundle UCL, radial collateral ligament (RCL), annular ligament (AL), and lateral ulnar collateral ligament (LUCL)] was measured on the PACS workstation with measurements obtained to the nearest tenth of a millimeter. All measurements were made in the middle of the ligament in the planes: in the coronal plane for the anterior bundle of the UCL, the RCL, and the LUCL and in the axial plane for the posterior bundle of the UCL and the AL. These were chosen based on a prior study in order to be used as comparative data [5]. The postero-lateral plica was measured in three dimensions (medio-lateral dimension on coronal images and antero-posterior and cranio-caudal dimension on sagittal images).
Statistical analysis

Descriptive statistics were performed for qualitative and quantitative MR imaging features. For qualitative data, the proportion (frequency) of each observation was calculated. For the quantitative data, the ligament thickness and postero-lateral plica size were described with range, standard deviation, and 25th, 50th, 75th, and 90th percentile. Qualitative features were considered as ordinal variables, and inter-reader variation was calculated using linear non-weighted kappa. Interpretation was according to Landis and Koch [7]. Kappa values between 0 and 0.2 are considered slight agreement, between 0.21 and 0.4 fair agreement, between 0.41 and 0.6 moderate agreement, between 0.61 and 0.8 substantial agreement, and between 0.81 and 1 almost perfect agreement. The interclass coefficient (ICC) was used to assess observer reliability for quantitative features. For all the statistical tests, a threshold of 0.05 for the P-value was considered as statistically significant. STATA statistical software (version 10.0) was utilized to perform the analysis.

\section{Results}

Qualitative evaluation

The qualitative results are summarized in Tables 1, 2, 3 and 4. The inter-observer agreement between readers was moderate to perfect ( $\mathrm{k}$ from 0.47 to 1.00 ) except for common flexor tendon evaluation, which was fair $(k=0.37)$.

The following results are derived from the consensus reading.

Ligaments (Table 1): $48 \%(10 / 21)$ and $10 \%(2 / 21)$ of the subjects showed partial tears of the anterior (Fig. 1) and posterior bundle of the ulnar collateral ligament, respectively. None of the subjects showed partial and/or full thickness tears of the lateral ligament complex (RCL, AL, LUCL). High signal intensity was visible in $43 \%(9 / 21)$ of the anterior UCLs and in none of the posterior UCLs, ALs, RCLs, and LUCLs.

Tendons (Table 2): Tendinopathy was seen in $19 \%(4 / 21)$ of common extensors. Common flexors and anterior tendons (biceps, brachioradialis) were normal in all subjects.

Bones (Table 3): Small-medium osteophytes were seen in the posteromedial portion of the humeroulnar articulation in $33 \%(7 / 21)$, in the hinge portion of the humeroulnar articulation in $38 \%(8 / 21)$, and in the humeroradial articulation in $5 \%$ $(1 / 21)$. Bone marrow edema was present in $24 \%(5 / 21)$ of the pitchers in the following locations: 2 in the coronoid process, 1 in the radial head, 1 in the humeral trochlea, and 1 in the medial epicondyle (Fig. 2a, b). 
Table 1 Qualitative evaluation of elbow ligaments
Anterior UCL, anterior bundle of the ulnar collateral ligament; posterior UCL, posterior bundle of the ulnar collateral ligament; $R C L$, radial collateral ligament; $A L$, annular ligament; $L U C L$, lateral ulnar collateral ligament. Values are in percentage with absolute values in parentheses

Data are given as percentages with parenthetical values representing the number of subjects

\begin{tabular}{|c|c|c|c|c|}
\hline Parameter & Reader 1 & Reader 2 & Agreement & Kappa \\
\hline \multicolumn{4}{|l|}{ Anterior UCL integrity } & \multirow[t]{5}{*}{0.53} \\
\hline Intact & $57(12)$ & $48(10)$ & $52(11)$ & \\
\hline Partial thickness tear less than $50 \%$ & $19(4)$ & $19(4)$ & $19(4)$ & \\
\hline Partial thickness tear more than $50 \%$ & $24(5)$ & $33(7)$ & $29(6)$ & \\
\hline Full thickness tear & $0(0)$ & $0(0)$ & $0(0)$ & \\
\hline \multicolumn{4}{|l|}{ Anterior UCL signal intensity } & \multirow[t]{4}{*}{0.86} \\
\hline Normal signal intensity & $14(3)$ & $5(1)$ & $5(1)$ & \\
\hline Striation & $43(9)$ & $48(10)$ & $52(11)$ & \\
\hline High signal intensity & $43(9)$ & $48(10)$ & $43(9)$ & \\
\hline \multicolumn{4}{|l|}{ Posterior UCL integrity } & \multirow[t]{5}{*}{0.47} \\
\hline Intact & $90(19)$ & $80(17)$ & $90(19)$ & \\
\hline Partial thickness tear less than $50 \%$ & $0(0)$ & $14(3)$ & $5(1)$ & \\
\hline Partial thickness tear more than $50 \%$ & $5(1)$ & $5(1)$ & $5(1)$ & \\
\hline Full thickness tear & $0(0)$ & $0(0)$ & $0(0)$ & \\
\hline \multicolumn{4}{|l|}{ Posterior UCL signal intensity } & \multirow[t]{4}{*}{1.00} \\
\hline Normal signal intensity & $95(20)$ & $95(20)$ & $95(20)$ & \\
\hline Striation & $5(1)$ & $5(1)$ & $5(1)$ & \\
\hline High signal intensity & $0(0)$ & $0(0)$ & $0(0)$ & \\
\hline \multicolumn{4}{|l|}{ LUCL integrity } & \multirow[t]{5}{*}{0.90} \\
\hline Intact & $95(20)$ & $100(21)$ & $100(21)$ & \\
\hline Partial thickness tear less than $50 \%$ & $5(1)$ & $0(0)$ & $0(0)$ & \\
\hline Partial thickness tear more than $50 \%$ & $0(0)$ & $0(0)$ & $0(0)$ & \\
\hline Full thickness tear & $0(0)$ & $0(0)$ & $0(0)$ & \\
\hline \multicolumn{4}{|l|}{ LUCL signal intensity } & \multirow[t]{4}{*}{1.00} \\
\hline Normal signal intensity & $95(20)$ & $95(20)$ & $95(20)$ & \\
\hline Striation & $5(1)$ & $5(1)$ & $5(1)$ & \\
\hline High signal intensity & $0(0)$ & $0(0)$ & $0(0)$ & \\
\hline \multicolumn{4}{|l|}{$\mathrm{RCL}$ integrity } & \multirow[t]{5}{*}{1.00} \\
\hline Intact & $100(21)$ & $100(21)$ & $100(21)$ & \\
\hline Partial thickness tear less than $50 \%$ & $0(0)$ & $0(0)$ & $0(0)$ & \\
\hline Partial thickness tear more than $50 \%$ & $0(0)$ & $0(0)$ & $0(0)$ & \\
\hline Full thickness tear & $0(0)$ & $0(0)$ & $0(0)$ & \\
\hline \multicolumn{4}{|l|}{ RCL signal intensity } & \multirow[t]{4}{*}{1.00} \\
\hline Normal signal intensity & $95(20)$ & $95(20)$ & $95(20)$ & \\
\hline Striation & $5(1)$ & $5(1)$ & $5(1)$ & \\
\hline High signal intensity & $0(0)$ & $0(0)$ & $0(0)$ & \\
\hline \multicolumn{4}{|l|}{ AL integrity } & \multirow[t]{5}{*}{1.00} \\
\hline Intact & $100(21)$ & $100(21)$ & $100(21)$ & \\
\hline Partial thickness tear less than $50 \%$ & $0(0)$ & $0(0)$ & $0(0)$ & \\
\hline Partial thickness tear more than $50 \%$ & $0(0)$ & $0(0)$ & $0(0)$ & \\
\hline Full thickness tear & $0(0)$ & $0(0)$ & $0(0)$ & \\
\hline \multicolumn{4}{|l|}{ AL signal intensity } & \multirow[t]{4}{*}{1.00} \\
\hline Normal signal intensity & $100(21)$ & $100(21)$ & $100(21)$ & \\
\hline Striation & $0(0)$ & $0(0)$ & $0(0)$ & \\
\hline High signal intensity & $0(0)$ & $0(0)$ & $0(0)$ & \\
\hline
\end{tabular}

Cartilage (Table 3): Diffuse cartilage lesions of the humeroulnar joint were detected in $14 \%(3 / 21)$.

Cubital tunnel (Table 4): The ulnar nerve was hyperintense in $81 \%(17 / 21)$ at the level of the cubital tunnel (Fig. 4) and had a flattened configuration in $38 \%(8 / 21)$. The cubital retinaculum was absent in $14 \%(3 / 21)$ and thick in $21 \%$ (4/21) (Fig. 3a, b).

Joint fluid (Table 4): No large effusions noted. One subject $[5 \%(1 / 21)]$ showed intra-articular bodies in the anterior and posterior recesses (Fig. 4). 
Table 2 Qualitative evaluation of elbow tendons

\begin{tabular}{lcccc}
\hline Parameters & Reader 1 & Reader 2 & Agreement & Kappa \\
\hline Biceps tendon & & & & 1.00 \\
$\quad$ Normal & $100(21)$ & $100(21)$ & $100(21)$ & \\
Tendinopathy & $0(0)$ & $0(0)$ & $0(0)$ & \\
Partial thickness tear & $0(0)$ & $0(0)$ & $0(0)$ & \\
Full thickness tear & $0(0)$ & $0(0)$ & $0(0)$ & \\
Common extensor tendon & & & & 0.64 \\
Normal & $67(14)$ & $62(13)$ & $71(15)$ & \\
Tendinopathy & $24(5)$ & $19(4)$ & $19(4)$ & \\
Partial thickness tear & $10(2)$ & $19(4)$ & $10(2)$ & \\
Full thickness tear & $0(0)$ & $0(0)$ & $0(0)$ & \\
Common flexor tendon & & & & 0.37 \\
Normal & $81(17)$ & $95(20)$ & $95(20)$ & \\
Tendinopathy & $14(3)$ & $0(0)$ & $0(0)$ & \\
Partial thickness tear & $5(1)$ & $5(1)$ & $5(1)$ & \\
Full thickness tear & $0(0)$ & $0(0)$ & $0(0)$ & \\
\hline
\end{tabular}

Data are given as percentages with parenthetical values representing the number of subjects

Olecranon fat pad (Table 4): Chronic changes in the olecranon fat pad were present in $33 \%(7 / 21)$ of the pitchers, and no subject showed signs of acute changes (low signal intensity on T1-weighted sequences and high signal intensity on fluidsensitive sequences) of the olecranon fat pad. (Fig. 5a, b).

\section{Quantitative evaluation}

The results from both readers are summarized in Table 5 .

The following results are from reader 2 . The median ligament thicknesses (with lower and upper ranges) were: anterior bundle of the UCL $4.6 \mathrm{~mm}(2.2-9.1 \mathrm{~mm})$, posterior bundle of the UCL $1.8 \mathrm{~mm}(0.6-2.9 \mathrm{~mm})$, RCL $1.9 \mathrm{~mm}(1.4-4.8 \mathrm{~mm})$, lateral UCL $2.5 \mathrm{~mm}(1.5-3.8 \mathrm{~mm})$, and AL $0.7 \mathrm{~mm}(0.5-$ $1.4 \mathrm{~mm}$ ). The median size of the postero-lateral plica (with lower and upper ranges) was antero-posterior $5.3 \mathrm{~mm}(3.8$ $7.1 \mathrm{~mm})$ by cranio-caudal $2.2 \mathrm{~mm}(1.5-4.3 \mathrm{~mm}) \times 2.7 \mathrm{~mm}$ medio lateral $(1.6-4.7 \mathrm{~mm})$.

\section{Discussion}

MR imaging is frequently the diagnostic modality of choice for evaluation of elbow internal derangements. MR imaging examinations are increasingly performed with 3-T magnets. Before contract signing and after a detailed physical examination, MR imaging of the elbow may be part of the diagnostic workup of professional baseball pitchers and other throwing athletes. Elbow MR imaging may also be obtained when patients have been injured during sports. Therefore, it can be important to know the baseline features of the elbow
Table 3 Qualitative evaluation of bone (osteophytes), bone marrow edema, and cartilage

\begin{tabular}{|c|c|c|c|c|}
\hline Parameters & Reader 1 & Reader 2 & Agreement & Kappa \\
\hline \multicolumn{4}{|c|}{ Postero-medial olecranon ostephytes } & \multirow[t]{5}{*}{0.68} \\
\hline None & $76(16)$ & $67(14)$ & $67(14)$ & \\
\hline Small & $19(4)$ & $24(5)$ & $24(5)$ & \\
\hline Medium & $5(1)$ & $10(2)$ & $10(2)$ & \\
\hline Large & $0(0)$ & $0(0)$ & $0(0)$ & \\
\hline \multicolumn{4}{|c|}{ Humero-ulnar osteophytes } & \multirow[t]{5}{*}{0.58} \\
\hline None & $57(12)$ & $57(12)$ & $62(13)$ & \\
\hline Small & $29(6)$ & $33(7)$ & $29(6)$ & \\
\hline Medium & $10(2)$ & $5(1)$ & $5(1)$ & \\
\hline Large & $5(1)$ & $5(1)$ & $5(1)$ & \\
\hline \multicolumn{4}{|c|}{ Humero-radial osteophytes } & \multirow[t]{5}{*}{1.00} \\
\hline None & $95(20)$ & $95(20)$ & $95(20)$ & \\
\hline Small & $0(0)$ & $0(0)$ & $0(0)$ & \\
\hline Medium & $0(0)$ & $0(0)$ & $0(0)$ & \\
\hline Large & $5(1)$ & $5(1)$ & $5(1)$ & \\
\hline \multicolumn{4}{|c|}{ Proximal radio-ulnar joint osteophytes } & \multirow[t]{5}{*}{1.00} \\
\hline None & $100(21)$ & $100(21)$ & $100(21)$ & \\
\hline Small & $0(0)$ & $0(0)$ & $0(0)$ & \\
\hline Medium & $0(0)$ & $0(0)$ & $0(0)$ & \\
\hline Large & $0(0)$ & $0(0)$ & $0(0)$ & \\
\hline \multicolumn{4}{|c|}{ Humero-ulnar cartilage } & \multirow[t]{4}{*}{0.71} \\
\hline Normal & $86(18)$ & $76(16)$ & $86(18)$ & \\
\hline Focal lesion & $5(1)$ & $10(2)$ & $0(0)$ & \\
\hline Diffuse lesion & $10(2)$ & $14(3)$ & $14(3)$ & \\
\hline \multicolumn{4}{|c|}{ Humero-radial cartilage } & \multirow[t]{4}{*}{0.86} \\
\hline Normal & $90(19)$ & $100(21)$ & $100(21)$ & \\
\hline Focal lesion & $0(0)$ & $0(0)$ & $0(0)$ & \\
\hline Diffuse lesion & $10(2)$ & $0(0)$ & $0(0)$ & \\
\hline \multicolumn{4}{|c|}{ Proximal radio-ulnar joint cartilage } & \multirow[t]{4}{*}{1.00} \\
\hline Normal & $100(21)$ & $100(21)$ & $100(21)$ & \\
\hline Focal lesion & $0(0)$ & $0(0)$ & $0(0)$ & \\
\hline Diffuse lesion & $0(0)$ & $0(0)$ & $0(0)$ & \\
\hline \multicolumn{4}{|c|}{ Bone marrow edema } & \multirow[t]{3}{*}{1.00} \\
\hline Absent & $76(16)$ & $76(16)$ & $76(16)$ & \\
\hline Present & $24(5)$ & $24(5)$ & $24(5)$ & \\
\hline
\end{tabular}

Data are given as percentages with parenthetical values representing the number of subjects

structures of non-symptomatic pitchers to avoid downstream false-positive reports with potentially important medical and financial consequences. These false-positive reports may actually be true lesions but are old and no longer symptomatic or clinically relevant. We frequently found elbow MR imaging abnormalities of the ligaments, tendons, and nerves, and less commonly in the joint articular cartilage, olecranon fat pad, and bones.

Three-Tesla MR imaging has a substantially higher SNR compared to lower field MR imaging (theoretically twice as much signal as 1.5-T systems), which facilitates high- 
Table 4 Qualitative evaluation of the ulnar nerve, retinaculum, joint effusion, loose bodies, and olecranon fat pad

\begin{tabular}{lcccc}
\hline Ulnar nerve signal intensity & & & & 0.72 \\
$\quad$ Normal & $19(4)$ & $14(3)$ & $19(4)$ & \\
Slightly hyperintense & $38(8)$ & $38(8)$ & $29(6)$ & \\
Moderate hyperintense & $33(7)$ & $38(8)$ & $43(9)$ & \\
Highly hyperintense & $10(2)$ & $10(2)$ & $10(2)$ & \\
Ulnar nerve shape & & & & 0.60 \\
Ovoid & $57(12)$ & $67(14)$ & $62(13)$ & \\
Flat & $53(9)$ & $33(7)$ & $38(8)$ & \\
Retinaculum & & & & 0.59 \\
Absent & $10(2)$ & $19(4)$ & $14(3)$ & \\
Present & $57(12)$ & $57(12)$ & $67(14)$ & \\
Thick & $33(7)$ & $24(5)$ & $19(4)$ & \\
Joint fluid (effusion) & & & & 0.69 \\
None & $71(15)$ & $57(12)$ & $67(14)$ & \\
Small & $29(6)$ & $43(9)$ & $33(7)$ & \\
Large & $0(0)$ & $0(0)$ & $0(0)$ & \\
Loose bodies & & & & 1.00 \\
Absent & $95(20)$ & $95(20)$ & $95(20)$ & \\
Present & $5(1)$ & $5(1)$ & $5(1)$ & \\
Olecranon fat pad & & & & 0.77 \\
Normal & $71(15)$ & $71(15)$ & $67(14)$ & \\
Acute & $0(0)$ & $0(0)$ & $0(0)$ & \\
Chronic & $29(6)$ & $29(6)$ & $33(7)$ & \\
\hline
\end{tabular}

Data are given as percentages with parenthetical values representing the number of subjects

resolution imaging with increased in-plane spatial resolution and thinner slices $[4,8,9]$. Furthermore, options such as SPAIR offer the possibility to have more homogeneous offisocenter fat suppression over chemically selective fat saturation, as is the case in our study population that was imaged in supine position with the elbow off the isocenter. It has been

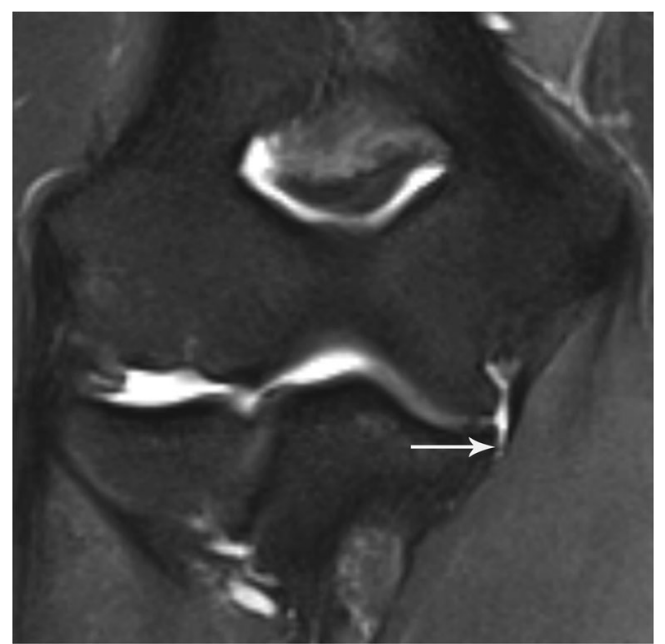

Fig. 1 A 20-year-old baseball pitcher: IW (TR 4,500/TE $46 \mathrm{~ms}$ ) coronal fat-suppressed sequences of the right elbow show distal articular-sided partial tear (arrow) of the anterior bundle of the ulnar collateral ligament ("T-sign")
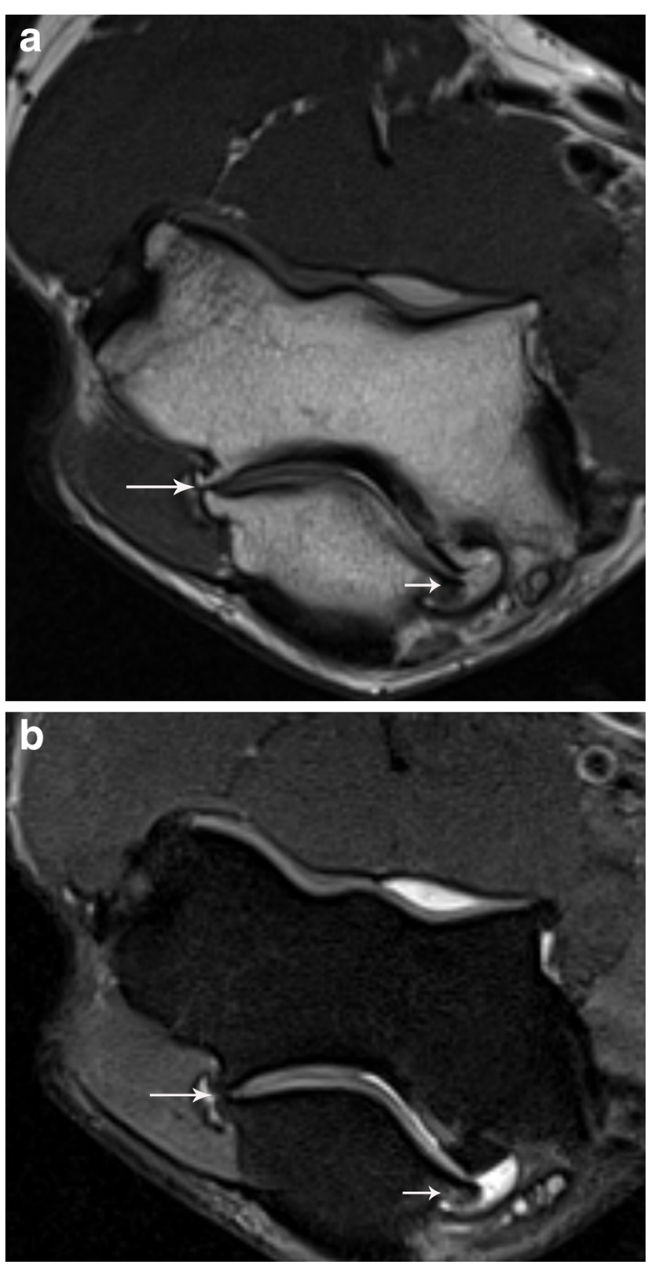

Fig. 2 A 32-year-old baseball pitcher (a) IW (TR 3,000/TE 43 ms) axial sequences and (b) IW (TR 3000/TE $45 \mathrm{~ms}$ ) fat-suppressed axial sequences show posterolateral (long arrow) and posteromedial (short arrow) osteophytes with bone marrow edema at the medial site

demonstrated that some musculoskeletal anatomical structures in the wrist such as the triangular fibrocartilage complex and scapholunate ligament are better visible on 3-T MR than on 1.5-T MR imaging [10, 11].

The anatomy of the elbow is also somewhat complex with small bundles such as the posterior UCL and complex anatomy such as the LUCL and could take advantage of high field MR imaging. To the best of our knowledge, there are no articles on 3-T MR imaging of the elbow in baseball pitchers. Husarik and colleagues reported the measurement of the collateral ligaments and of the posterior and postero-lateral plica in 60 asymptomatic non-pitcher non-professional athlete subjects using 1.5-T MR. We performed the measurements in the same plane and in the same locations as described by Husarik et al. [5]. We found substantial differences in the thickness of the anterior and posterior ulnar collateral ligaments, whereas we did not record any substantial differences in the lateral collateral ligament structures. In our study, the median thickness was $4.6 \mathrm{~mm}$ (compared to $2.5 \mathrm{~mm}$ ) for the anterior 

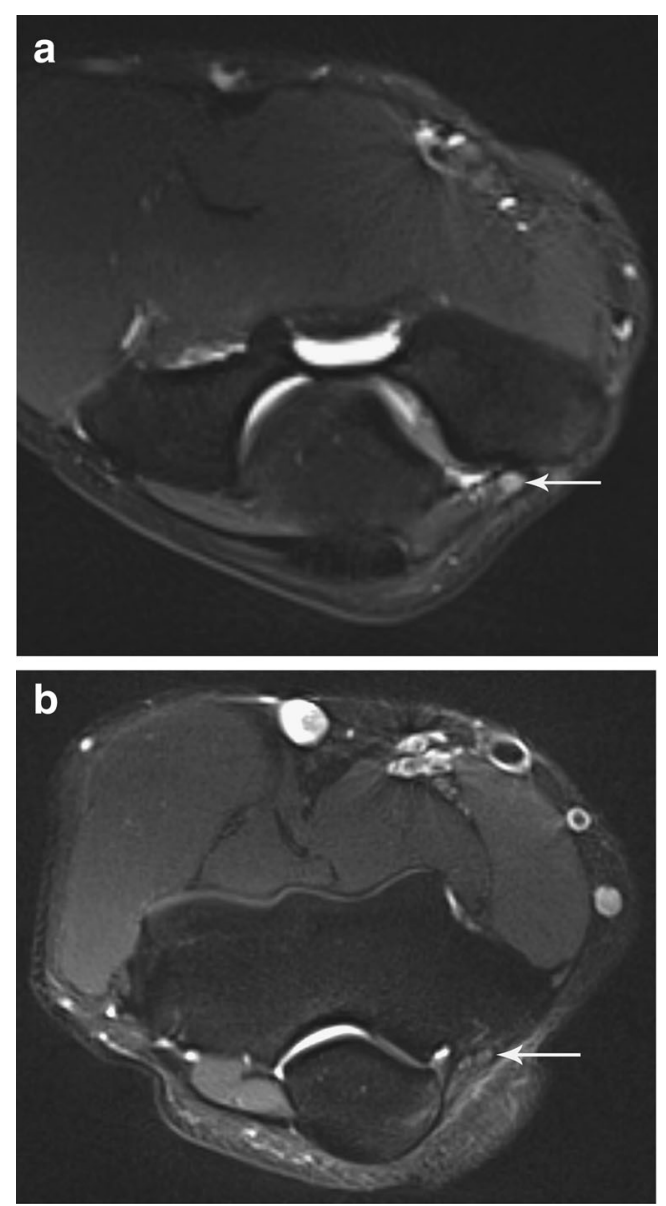

Fig. 3 a An 18-year-old male baseball pitcher. IW (TR 3,270/TE 72 ms) axial fat-suppressed (SPAIR) sequence of the right elbow shows moderate hyperintensity (brighter than muscle but less than fluid) of the ulnar nerve (arrow) at the level of the cubital tunnel. b A 20-year-old male baseball pitcher. IW (TR 3,270/TE 72) axial fat-suppressed (SPAIR) sequence of the right elbow shows isointensity (compared to the muscle) of the ulnar nerve (arrow)

bundle UCL and $1.8 \mathrm{~mm}$ (compared to $1 \mathrm{~mm}$ ) for the posterior bundle UCL. The pathophysiologic mechanism of pitching helps to explain these results. The biomechanics of pitching can be divided into five phases: wind up, cocking, acceleration, deceleration, and follow-through [2]. In the late cocking phase, the maximal valgus forces are applied to the medial elbow $[2,3,12]$. Micro-trauma and shearing forces in the medial compartment lead to injury of the ulnar collateral ligament. These injuries are well documented on MR imaging of symptomatic throwing athletes [13-18]. MR imaging abnormalities of the ligaments have been described in nonsymptomatic baseball pitchers as well. Kooima et al. studied 16 non-symptomatic baseball players (12 pitchers and 4 position players) and found that $87 \%$ of the players had abnormalities such as thickening, heterogeneity, and discontinuity of the UCL [1]. Hurd et al. found similar results on MR of asymptomatic high school age baseball pitchers from 15 to 19 years old; they found a thickened anterior bundle of the

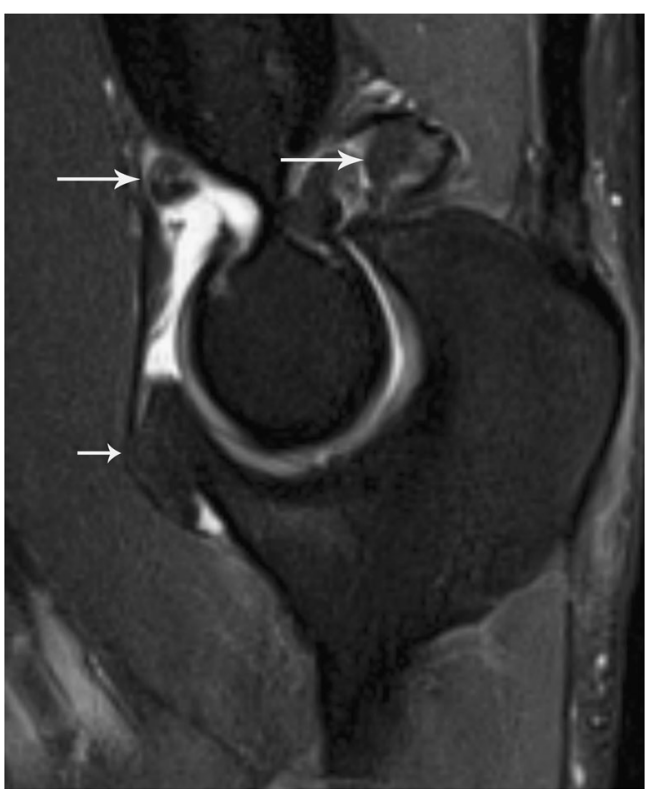

Fig. 4 A 32-year-old baseball pitcher. IW (TR 4,360/42 ms) sagittal fatsuppressed sequence shows intra-articular bodies (long arrows) in anterior and posterior recesses and osteophyte (short arrow) of the coronoid process of the ulna

UCL in $65 \%$ of the pitchers. Furthermore, the study confirmed the significant relationship between the UCL and posteromedial subchondral sclerosis [19]. However, these features have not been uniformly described in all pitchers. Different results were seen by Jazrawi et al. studying 14 asymptomatic young male pitchers less than 18 years old; they found only minimal change and no significant signal abnormalities in the UCL. The differences compared to other studies (including our data) may be due to the relatively short duration of pitching of the young baseball players in this study or the imaging technique used [20]. These prior investigations of elbow MR imaging of baseball players performed only qualitative evaluation of the collateral ligaments, were performed on 1.5-T MR imaging systems, and sometimes involved mixed populations of professional baseball players regardless of their role (pitchers and fielding positions). Our study involves only baseball pitchers examined on 3-T MR imaging with qualitative and quantitative analysis. We confirmed the thickening of the ulnar collateral ligament and high prevalence of partial tears (48\%) and higher signal intensity (43\%) of the anterior UCL. The posterior bundle of the UCL has not previously been described in non-symptomatic baseball pitchers and may relate to lower resolution techniques. In our study, it was substantially thicker than in the non-pitching population. This implies that it may play a more important role than previously thought or that baseball pitching generates unique biomechanics to induce this particular morphologic alteration.

Osteophytosis is a well-known consequence in throwing athletes, so it is not surprising that these were present in our 

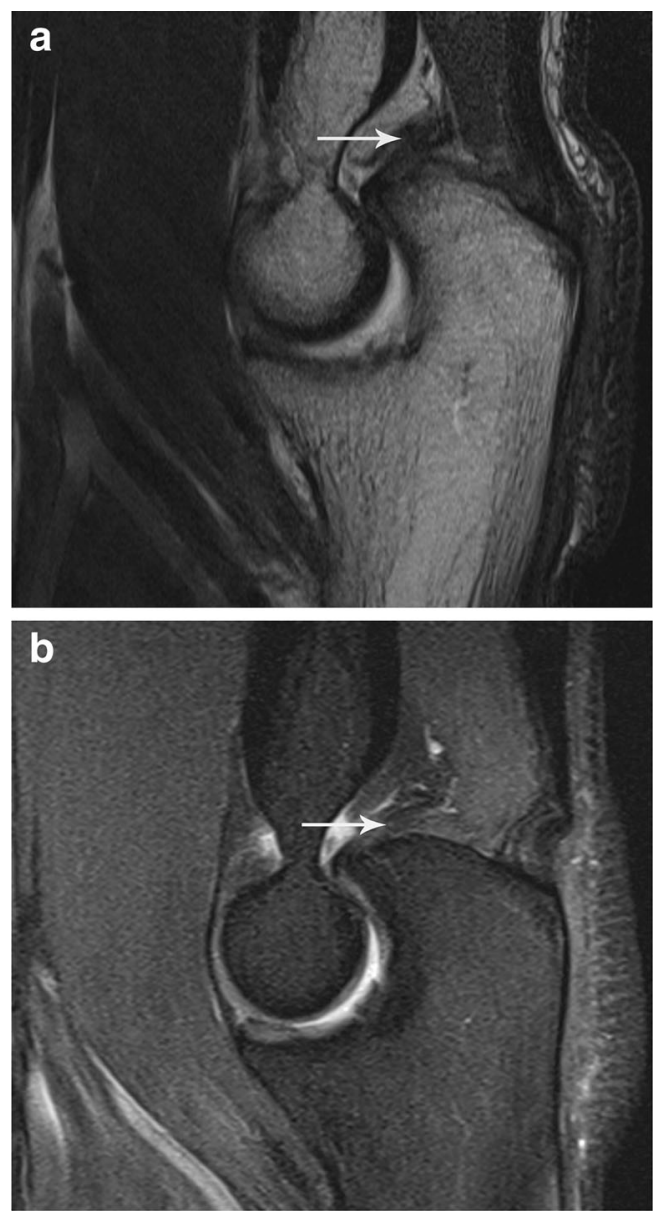

Fig. 5 A 20-year-old baseball pitcher. (a) IW (TR 4,170/TE 49 ms) sagittal and (b) IW (TR/TE 2,000/49 ms) sagittal fat-suppressed sequences of the right elbow show a region of low signal intensity changes on the olecranon fat pad compatible with fibrosis (arrow)

study group. The bone marrow edema that was noted could be related to subclinical contusions, stress reactions, or when in a subarticular location to early osteoarthritis.

In our study of baseball pitchers, we had a higher proportion of subjects with ulnar nerve signal alteration, $81 \%(17 /$ $21)$ compared to $60 \%(36 / 60)$ in the non-symptomatic nonpitchers population [21]. This might be explained with the pathophysiology of pitching; however, the results could be biased by high-resolution 3-T MR imaging.

The higher magnetic field strength may enhance the conspicuity of the nerve signal, perhaps even beyond the range of what is currently considered normal. In our study, we also used a very robust form of fat suppression, different from the spectral selective fat suppression used in the Husarik study for axial images [21]. Therefore, the positivity criteria for "abnormal" nerve signal may need to be reconsidered when employing 3-T MR imaging. In addition, some of the higher signal intensity of the nerve could be artifactual because of the magic angle phenomenon (MAP). Kästel et al. investigated the contribution of MAP to false-positive increases of
Table 5 Quantitative evaluation of ligaments and postero-lateral plica

\begin{tabular}{lccccccc}
\hline Parameters and readers & $25^{\text {th }}$ & $50^{\text {th }}$ & $75^{\text {th }}$ & $90^{\text {th }}$ & Range & SD & ICC \\
\hline Ant. UCL & & & & & & & \\
$\quad$ Reader 2 & 3.5 & 4.6 & 5.9 & 7.5 & $2.2-9.1$ & 1.90 & \\
$\quad$ Reader 3 & 3.4 & 4.6 & 6.5 & 8.5 & $2.4-9.8$ & 2.17 & 0.951 \\
Post. UCL & & & & & & & \\
$\quad$ Reader 2 & 1.0 & 1.8 & 2.2 & 2.5 & $0.6-2.9$ & 0.70 & \\
$\quad$ Reader 3 & 1.2 & 1.3 & 1.6 & 2.1 & $0.8-2.8$ & 0.53 & 0.808 \\
RCL & & & & & & & \\
$\quad$ Reader 2 & 1.7 & 1.9 & 1.9 & 2.9 & $1.4-4.8$ & 0.76 & \\
$\quad$ Reader 3 & 1.6 & 1.8 & 2.0 & 2.8 & $0.9-5.5$ & 0.97 & 0.686 \\
LUCL & & & & & & & \\
$\quad$ Reader 2 & 2.15 & 2.5 & 3.2 & 3.7 & $1.5-3.8$ & 0.64 & \\
$\quad$ Reader 3 & 1.75 & 2 & 2.5 & 3.0 & $1.1-3.5$ & 0.57 & 0.448 \\
AL & & & & & & & \\
$\quad$ Reader 2 & 0.6 & 0.7 & 0.8 & 1 & $0.5-1.4$ & 0.26 & \\
$\quad$ Reader 3 & 0.9 & 1 & 1.15 & 1.4 & $0.7-1.4$ & 0.19 & 0.479 \\
Postero-lat plica cranio-caudal & & & & & & \\
$\quad$ Reader 2 & 1.9 & 2.2 & 2.8 & 3.1 & $1.5-4.3$ & 0.69 & \\
$\quad$ Reader 3 & 2.5 & 2.9 & 3.3 & 4.1 & $1.6-4.3$ & 0.76 & 0.819 \\
Postero-lat plica medio-lateral & & & & & & \\
$\quad$ Reader 2 & 2.5 & 2.7 & 3.2 & 3.6 & $1.6-4.7$ & 0.67 & \\
$\quad$ Reader 3 & 2.5 & 2.8 & 3.2 & 4.3 & $1.4-5.1$ & 0.90 & 0.589 \\
Postero-lat plica sagittal & & & & & & & \\
$\quad$ Reader 2 & 4.6 & 5.3 & 6.15 & 7.0 & $3.8-7.1$ & 0.93 & \\
$\quad$ Reader 3 & 5.1 & 5.4 & 6.35 & 7.3 & $4.1-7.3$ & 0.97 & 0.769 \\
\hline
\end{tabular}

Anterior UCL, anterior bundle of the ulnar collateral ligament; posterior $U C L$, posterior bundle of the ulnar collateral ligament; $R C L$, radial collateral ligament; $L U C L$, lateral ulnar collateral ligament; $A L$, annular ligament; $S D$, standard deviation; $I C C$, interclass correlation

Values of ligaments and postero-lateral plica are in millimeters

intraneural signal intensity of peripheral nerves on 3-T MRI [22]. The authors found that only at angle positions greater than $30^{\circ}$, the signal intensity of the nerve potentially increases more than $10 \%$. Our subjects were imaged in a wide-bore 70 $\mathrm{cm}$ magnet and were positioned with their elbows at their sides and positioned as near to iso-center as possible, body habitus allowing. The elbow "carrying angle" is typically 5 to $15^{\circ}$ away from the body. This is important because the angulation of the ulnar nerve at the level of the cubital tunnel will be substantially lower than $30^{\circ}$ relative to $\mathrm{B} 0$. The results of the study by Kästel et al. also showed that patients with peripheral neuropathy exposed to an angle position of $55^{\circ}$ (maximum MAP expected) showed higher intraneural signal intensity compared to the control group. Therefore, according to their results, the potential of the MAP to be responsible for false-positive intraneural increase of signal intensity is low. However, MAP and mild neuropathy are both associated with only a minor increase in intraneural T2-weighted signal intensity. Therefore, subjects that have mild increased signal could 
represent a technical issue. However, moderate or marked increases of signal in subjects are probably not due to technical issues and represent pathological alterations of the ulnar nerve, albeit subclinical in this non-symptomatic population.

Interestingly, none of the subjects in our study population showed acute changes in the olecranon fat pad but had presumptive features of chronic scarring. This region of fibroticappearing material may have downstream relevance contributing to the posterior/posteromedial impingement syndrome that some throwing athletes develop [23].

Our retrospective study has some limitations. First, the study population is relatively small. Second, the readers were aware of the fact that the players were asymptomatic. Third, there was not a comparison group. Fourth, all the included pitchers were claiming to be non-symptomatic, but we cannot exclude that some pitchers may dismiss symptoms before contract signing.

In summary, our study found a high prevalence of 3-T MR imaging abnormalities of the ligaments, tendons, bones, and olecranon fat pad in the elbow of non-symptomatic major league baseball pitchers during the diagnostic workup before contract signing. None of our subjects showed acute changes in the olecranon fat pad, full thickness tendon tears, or large joint effusions. Therefore, the radiologist and orthopedist should be aware of these features when interpreting 3-T elbow MR imaging of baseball pitchers.

Conflict of interest No conflict of interest.

\section{References}

1. Kooima CL, Anderson K, Craig JV, Teeter DM, van Holsbeeck M. Evidence of subclinical medial collateral ligament injury and posteromedial impingement in professional baseball players. Am J Sports Med. 2004;32(7):1602-6.

2. Pappas AM, Zawacki RM, Sullivan TJ. Biomechanics of baseball pitching. A preliminary report. Am J Sports Med. 1985;13(4):216-22.

3. Fleisig GS, Andrews JR, Dillman CJ, Escamilla RF. Kinetics of baseball pitching with implications about injury mechanisms. Am J Sports Med. 1995;23(2):233-9.

4. Bolog N, Nanz D, Weishaupt D. Muskuloskeletal MR imaging at 3.0 T: current status and future perspectives. Eur Radiol. 2006;16(6): 1298-307.

5. Husarik DB, Saupe N, Pfirrmann CW, Jost B, Hodler J, Zanetti M. Ligaments and plicae of the elbow: normal MR imaging variability in 60 asymptomatic subjects. Radiology. 2010 Oct;257(1):185-94.
6. Chhabra A, Chalian M, Soldatos T, Andreisek G, Faridian-Aragh N, Williams E, et al. 3-T high-resolution MR neurography of sciatic neuropathy. AJR Am J Roentgenol. 2012 Apr;198(4):W357-64.

7. Landis JR, Koch GG. The measurement of observer agreement for categorical data. Biometrics. 1977 Mar;33(1):159-74.

8. Kuo R, Panchal M, Tanenbaum L, Crues JV. 3.0 Tesla imaging of the musculoskeletal system. J Magn Reson Imaging. 2007;25(2):24526.

9. Gandhi RT, Kuo R, Crues JV. Technical considerations and potential advantages of musculoskeletal imaging at 3.0 Tesla. Semin Musculoskelet Radiol. 2008;12(3):185-95.

10. Lenk S, Ludescher B, Martirosan P, Schick F, Claussen CD, Schlemmer HP. 3.0 T high-resolution MR imaging of carpal ligaments and TFCC. Rofo. 2004;176(5):664-7.

11. Saupe N, Prussmann KP, Luechinger R, Bosiger P, Marincek B, Weishaupt D. MR imaging of the wrist: comparison between 1.5and 3-T MR imaging - preliminary experience. Radiology. 2005 Jan;234(1):256-64.

12. Lynch JR, Waitayawinyu T, Hanel DP, Trumble TE. Medial collateral ligament injury in the overhand-throwing athlete. J Hand Surg [Am]. 2008 Mar;33(3):430-7.

13. Mirowitz SA, London SL. Ulnar collateral ligament injury in baseball pitchers: MR imaging evaluation. Radiology. 1992 Nov;185(2):5736.

14. Schwartz ML, Al-Zahrani S, Morwessel RM, Andrews JR. Ulnar collateral ligament injury in the throwing athlete: evaluation with saline-enhanced MR arthrography. Radiology. 1995;197(1):297-9.

15. Nakanishi K, Masatomi T, Ochi T, Ishida T, Hori S, Ikezoe J, et al. MR arthrography of elbow: evaluation of the ulnar collateral ligament of elbow. Skelet Radiol. 1996 Oct;25(7):629-34.

16. Ouellette H, Kassarjian A, Tetreault P, Palmer W. Imaging of the overhead throwing athlete. Semin Musculoskelet Radiol. 2005 Dec;9(4):316-33.

17. Ouellette H, Bredella M, Labis J, Palmer WE, Torriani M. MR imaging of the elbow in baseball pitchers. Skele Radiol. 2008 Feb;37(2):115-21.

18. Ouellette HA, Palmer W, Torriani M, Bredella MA. Throwing elbow in adults. Semin Musculoskelet Radiol. 2010 Sep;14(4):412-8.

19. Hurd WJ, Eby S, Kaufman KR, Murthy NS. Magnetic resonance imaging of the throwing elbow in the uninjured, high school-aged baseball pitcher. Am J Sports Med. 2011 Apr;39(4):722-8.

20. Jazrawi LM, Leibman M, Mechlin M, Yufit P, Ishak C, Schweitzer $\mathrm{M}$, et al. Magnetic resonance imaging evaluation of the ulnar collateral ligament in young baseball pitchers less than 18 years of age. Bull Hosp Jt Dis. 2006;63(3-4):105-7.

21. Husarik DB, Saupe N, Pfirrmann CW, Jost B, Hodler J, Zanetti M. Elbow nerves: MR findings in 60 asymptomatic subjects-normal anatomy, variants, and pitfalls. Radiology. $2009 \mathrm{Jul} ; 252(1): 148-56$.

22. Kastel T, Heiland S, Baumer P, Bartsch AJ, Bendszus M, Pham M. Magic angle effect: a relevant artifact in MR neurography at $3 \mathrm{~T}$ ? AJNR Am J Neuroradiol. 2011 May;32(5):821-7.

23. Cohen SB, Valko C, Zoga A, Dodson CC, Ciccotti MG. Posteromedial elbow impingement: magnetic resonance imaging findings in overhead throwing athletes and results of arthroscopic treatment. Arthroscopy. 2011 Oct;27(10):1364-70. 